\title{
miR-2954 Inhibits PI3K Signaling and Induces Autophagy and Apoptosis in Myocardium Selenium Deficiency
}

\author{
Qi Liu $\quad$ Jingzeng Cai ${ }^{\mathrm{a}} \quad$ Yunan Gao ${ }^{\mathrm{d}} \quad$ Jie Yang ${ }^{\mathrm{a}} \quad$ Yafan Gong ${ }^{\mathrm{a}}$ \\ Ziwei Zhang ${ }^{a, b, c}$
}

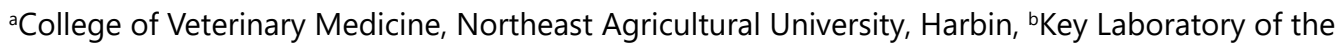
Provincial Education Department of Heilongjiang for Common Animal Disease Prevention and Treatment, College of Veterinary Medicine, Northeast Agricultural University, Harbin, Key Laboratory of Animal Cellular and Genetic Engineering of Heilongjiang Province, Northeast Agricultural University, Harbin, dDepartment of Cardiology, the Fourth Affiliated Hospital of Harbin Medical University, Harbin, China

\section{Key Words}

Selenium Deficiency • Myocardiosis • miR-2954 • Autophagy • Apoptosis • PI3K

\begin{abstract}
Background/Aims: Selenium (Se) deficiency can lead to several cardiac diseases, including Keshan disease in humans, mulberry heart disease in pigs and cardiac injury in chickens. MicroRNAs have been a research focus in recent years and have been shown to participate in a new avenue of cell death-autophagy, which can play a significant role in several types of heart disease. Methods: MicroRNAome analysis showed that the expression of miR-2954 was increased in the myocardium of selenium-deficient chickens, and PI3K was predicted to be the target gene. The target relationship between miR-2954 and PI3K was verified with a double fluorescence enzyme assay and RNA Protein Interaction Prediction and molecular docking software. qRT-PCR and western blotting were used to detect the expression of PI3K and related pathway components in selenium-deficient chickens and miR-2954 knockout/overexpression cardiomyocytes. Results: In this study, we observed that miR-2954 overexpression led to inhibition of PI3K pathway in vivo and in vitroled to inhibition of the PI3K pathway in vivo and in vitro. Conclusion: The expression of miR-2954 was increased in selenium-deficient myocardium, whereas overexpression of miR-2954 led to autophagy and apoptosis of myocardial cells during cardiac injury through regulation of the PI3K pathway; whether this phenomenon is a self-protection mechanism of the organism or damage caused by miR2954 requires further study. Our findings provides new insight apoptosis in cardiomyocytes; additionally, we aim to provide a new direction for the diagnosis and targeted treatment of myocardial diseases. Province, Northeast Agricultural University, Harbin 150030 (China), E-Mail zhangziwei@neau.edu.cn
\end{abstract}




\section{Cellular Physiology Cell Physiol Biochem 2018;51:778-792

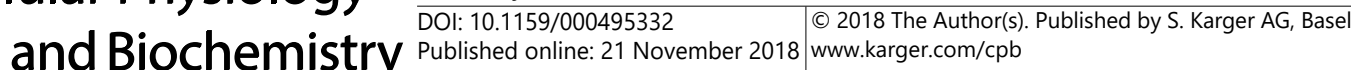 \\ Liu et al.: Role of miR-2954 in Se Deficiency Myocardium}

\section{Introduction}

Selenium (Se) has antioxidant, anti-inflammatory, chemo-preventive and antiviral properties that are essential for human and animal physiological health [1-3]. Additionally, Se deficiency can cause oxidative stress in chicken myoblasts [4], immune organs [5], and the liver, pancreas [6], and digestive system [7] and, according to our previous study, can induce oxidative stress in chicken cardiomyocytes [8]. Among the organs, the heart is a core organ in the body, and abnormal heart function leads to disease. Selenium deficiency is implicated in Keshan disease in humans and a degenerative white muscle disease in animals that affects both skeletal and cardiac muscles [9]. The first case of Keshan disease in humans was reported in 1937 during an epidemic in some rural areas of China, which was determined to be associated with Se deficiency. Additionally, changes in Se content can cause reversible myocardial injury in humans. In a study by Zhang, Se deficiency damaged mitochondrial structure and function in rats, leading to heart failure [10]. In previous studies, a close relationship between Se deficiency and cardiovascular disease has been demonstrated; however, the specific mechanism remains unknown. Many studies have shown that selenium deficiency is associated with myocardial apoptosis. Apoptosis is programmed cell death and is an active cell death process controlled by genes, accompanied by early degradation of DNA, alteration of chromatin concentration, cell atrophy and fragmentation. Cardiomyocytes are terminal differentiated cells and can induce remodeling through adaptation to various stresses, such as ischemia reperfusion, heart failure, and aging, via apoptosis, resulting in the loss of numerous myocardial cells. Cardiomyocytes apoptosis may cause damage to the myocardium and permanent dysfunction. In a previous study, we successfully established a model of Se deficient myocardial injury in chickens and confirmed that Se deficiency can lead to apoptosis in the heart.

MicroRNAs (miRNAs) are short noncoding RNA molecules that regulate gene expression posttranscriptionally. miRNAs also play an important role in the development of heart and liver diseases based on recent seminal papers and reviews. In a study by Yan MJ with rats, miR-31a-5p was found to protects myocardial cells against apoptosis by targeting Tp53. Jeong et al. indicate that miR-25 TuD (Tough Decoy) is an effective long-term suppressor of miR25 and a promising therapeutic candidate to treat heart failure in humans [11]. According to Verjans, heart failure-associated down regulation of the miRNA-221/222 family enables fibrosis signaling in a pressure-overloaded heart in rats [12]. In our previous study, we showed in chickens that regulation of miR-200a-5p and its target gene blocks necroptosis in the heart, which revealed a novel myocardial necrosis regulation model in heart disease [13].

In this study, we focused on miR-2954, and the avian gene for miR-2954 is on the Z sex chromosome. According to Lin et al., miR-2954, but not other Z-linked miRNAs, preferentially targets $\mathrm{Z}$ chromosome-encoded genes, including several genes that are expressed in a sexually dimorphic manner in the zebra finch brain. The expression of miR-2954 was also found in a second location, immediately below the liver. As shown in previous studies, Se deficiency can lead to cardiac injury; however, the exact mechanism remains unclear. Based on microRNAome analysis, Se deficiency causes changes in the expression of miR-2954, and in this study, we focused on confirming target gene and downstream pathways regulated by miR-2954. A microRNAome analysis was conducted, and then miRDB (http://www. mirdb.org/) and TargetScan (http://www.targetscan.org/mamm_31/), two online miRNA target gene prediction websites, both showed that PI3K could be a target gene of miR-2954. RNA-Protein Interaction Prediction and molecular docking software (Hex8.0.0 and PyMOL) also confirmed this result. Our present study was designed to verify PI3K as the target gene of miR-2954 and to further investigate the mechanism of myocardial injury induced by selenium deficiency in vivo and in vitro.

PI3Ks are a family of lipid kinases that integrate signals from growth factors, cytokines, and other environmental cues and translate them into intracellular signals that regulate multiple signaling pathways. These pathways control many physiological functions and cellular processes, including cell proliferation, growth, survival, motility, and metabolism. 
The PI3K/AKT pathway is widely considered an essential signaling pathway that controls autophagy, metabolism and oxidative stress. Thus, the PI3K/AKT signaling pathway could be a therapeutic target under certain oxidative stress conditions, such as the aging process and autophagy.

In a previous study, we successfully established a model of Se deficiency-induced myocardial injury in chickens and demonstrated that Se deficiency can lead to apoptosis in the heart. Based on current understanding, we decided to focus on the role of miR-2954 in Se deficiency in chickens and cardiomyocytes. The present study was designed to understand whether miR-2954 affects autophagy and apoptosis in cardiomyocytes. Accordingly, we developed a Se-deficient chicken model to observe the effects of a miR-2954 mimic and inhibitor on cardiac tissue through the PI3K/AKT pathway to further explore the role of miR-2954 in cardiomyocytes and selenium deficient tissues.

\section{Materials and Methods}

Replication of Se deficiency muscular dystrophy chicken model

One hundred and eighty one-day-old chickens (Weiwei Co. Ltd., Harbin, China) were randomly divided into two groups, Control and Low-Se (90 chickens/group). Each group had three replicates with thirty chickens per replicate. Five random samples in each group were used for miRNA sequencing, ten for realtime quantitative PCR, ten for Western blot and five for electron microscopy. The in vivo treatment groups were maintained on a Se-sufficient diet (C group) containing $0.23 \mathrm{mg} / \mathrm{kg}$, or a Se-deficient diet (L group) containing $0.008 \mathrm{mg} / \mathrm{kg}$ for 25 days. Our group previously described the Se-deficient diet composition and the detailed process used to establish the Se-deficiency model. Tissues were frozen immediately with Trizol and stored at $-80^{\circ} \mathrm{C}$ until required.

\section{MicroRNAome analysis and prediction of target genes}

Total RNA was extracted from the myocardial tissues of the control and Se-deficient groups and was processed according to the manufacturer's instructions (Novogene Bioinformatics Technology Co, Ltd.). After quantification and qualification of the total RNA, the RNA was used as input material for synthesis of microRNA libraries. Sequencing libraries were prepared using a NEBNext ${ }^{\circ}$ Multiplex Small RNA Library Prep Set for Illumina ${ }^{\circledR}$ (NEB, USA) following the manufacturer's recommendations. Bioinformatics analysis of the differential data from the control and Se-deficient groups was performed using the DESeq R package. The prediction of target genes was achieved using two online prediction websites, miRDB (http://www. mirdb.org/) and TargetScan (http://www.targetscan.org/mamm_31/).

\section{Sections for electron microscopy}

The technique adopted to observe ultrastructural changes was similar to that used in our previous study. The cardiac tissues (size: $1.0 \mathrm{~mm} \times 1.0 \mathrm{~mm} \times 1.0 \mathrm{~mm}$ ) were fixed immediately in $2.5 \%$ glutaraldehydephosphate-buffered saline (v/v, pH 7.2), postfixed in 1\% osmium tetroxide (v/v) and stained with $4.8 \%$ uranyl acetate following dehydration. The samples were washed in propylene oxide and impregnated with epoxy resins. The semifine sections were contrasted with uranyl acetate and lead citrate for microscopic observation. Microphotographs were taken with a transmission electron microscope (GEM1200ES, Japan).

\section{Primary cardiomyocyte culture}

Primary cardiomyocytes were isolated from 12-day-old chicken embryos for culture. The skin surface was disinfected using 75\% alcohol, and the chest dissected to gather the apical portion of the pericardium (approximately $1 / 3$ of the heart), which was transferred into PBS (stored at $4^{\circ} \mathrm{C}$ and warmed to $37^{\circ} \mathrm{C}$ before the experiments). Tissues were washed to remove fat, connective tissues and blood clots. Later, the myocardial tissue was cut into small pieces and washed 3 times PBS. Then, enzymatic digestion was conducted with collagenase II (Invitrogen, Carlsbad, CA,USA) for $15 \mathrm{~min}$ in a constant temperature magnetic stirrer $\left(37^{\circ} \mathrm{C}, 100 \mathrm{r} / \mathrm{min}\right)$ for assimilation, followed by centrifugation, and an equal volume of DMEM/F12 (GIBCO, Grand Island, NY, USA) containing 10\% fetal bovine serum (Thermo Fisher, USA) and 


\section{Cellular Physiology Cell Physiol Biochem 2018;51:778-792 and Biochemistry \begin{tabular}{l|l} 
DOI: 10.1159/000495332 & $\begin{array}{l}\text { () } 2018 \text { The Author(s). Published by S. Karger AG, Basel } \\
\text { www.karger.com/cpb }\end{array}$
\end{tabular}}

Liu et al.: Role of miR-2954 in Se Deficiency Myocardium

$1 \times$ myocillin (Solarbio, China) was added to terminate digestion. Tissues were redigested until the small tissue fragments were completely digested. All supernatant components were collected with 300 mesh and 500 mesh filters. The cell suspension was centrifuged at $600 \mathrm{rpm}$ for $5 \mathrm{~min}$ and resuspended in DMEM/F12 two times (first for $1 \mathrm{~h}$ and then for $1.5 \mathrm{~h}$ ) and then placed in disposable Petri dishes for differential adhesion (Thermo Fisher,USA). Finally, non-adherent cells (cardiomyocytes) were collected, centrifuged at $600 \mathrm{rpm}$, counted, plated in 6-well plates at $3 \times 10^{5}$ cell/well and incubated at $37^{\circ} \mathrm{C}$ with $95 \%$ air and $5 \% \mathrm{CO} 2$ in an adherent culture box for $48 \mathrm{~h}$. For each group, 3 replicates were prepared with $6 \times 10^{5}$ cardiomyocytes per replicate. For luciferase activity detection, 4 replicates were prepared for each group. For fluorescence detection, 3 repeats were prepared for each group and 3 repeats were used during real-time quantitative PCR. Western blotting was performed using 3 repeats, and flow cytometry analysis was conducted for 4 replicates in each group.

\section{Cell transfection with miR-2954 mimic and miR-2954 inhibitor}

Synthetic, chemically modified short RNA oligonucleotides were purchased from Shanghai Gene Pharma Co. Ltd. The miR-2954 mimic sequence used in the study was 5'-CAUCCCCAUUCCACUCCUAGCA-3', and the negative control 5'-UUUGUACUACACAAAAGUACUG-3', respectively. The miR-2954 inhibitor and inhibitor negative control (inhibitor-NC) sequences were 5'-UGCUAGGAGUGGAAUGGGGAUG-3' (m represents 2'-Ome) and 5'-CAGUACUUUUGUGUAGUACAAA-3', respectively. Cells were transfected with the miR-2954-mimic, mimic-NC, inhibitor and inhibitor-NC at $100 \mathrm{nM}$. The transfection reactions were performed using the Lipofectamine 2000 reagent (Invitrogen) in Opti-MEM medium according to the manufacturer's instruction.

Preparation of the PI3K 3'UTR luciferase construct and the luciferase activity assay

The pMIR-REPORT plasmids for the miR-2954 target PI3K 3'UTR were constructed as wild-type (WT) pMIR-PI3K of miR-2954 response elements from PI3K 3'UTR or mutant (MUT) pMIR-PI3K. The sequences of the single-stranded oligo pairs were used to generate the pMIR-PI3K constructs (WT and MUT). The oligonucleotides were then annealed and inserted into the pMIR-REPORT vector (Thermo Fisher). The empty pMIR-REPORT vector was used as the control. Cells were cotransfected with the pMIR-PI3K-WT or pMIR-PI3K-MUT plasmid constructs and miR-mimic using Lipofectamine 2000 (Invitrogen) according to the manufacturer's protocol. Twenty-four hours after transfection, the medium in culture plates was changed, and luciferase activity was determined using a Dual-Luciferase Reporter Assay System (Promega).

\section{RNA-Protein Interaction Prediction(RPISeq)}

RNA-protein interactions (RPIs) play important roles in a wide variety of cellular processes. Highthrough put experiments to identify RNA-protein interactions are beginning to provide valuable information about the complexity of RNA-protein interaction networks but are expensive and time consuming. RPISeq was developed to address the requirement for reliable computational methods for predicting RNA-protein interactions. RPISeq is a family of machine learning classifiers for predicting RNA-protein interactions using only sequence information. In the present study, we used RPISeq to predict the interaction between miR2954 and the target gene PI3K.

\section{Molecular docking and analysis software to analyze interactions between miR-2954 and PI3K}

Hex8.0.0 is an interactive molecular graphics program for calculating and displaying feasible docking modes of protein and DNA molecules pairs. The program is the only docking and superposition program to use spherical polar Fourier (SPF) correlations to accelerate the calculations. PyMOL (Schrödinger) is a usersponsored molecular visualization system on an open-source foundation maintained and distributed by Schrodinger and is suitable for making high-quality three-dimensional structure images of small molecules or biological macromolecules.

\section{Detection of autophagosomes via fluorescence detection}

Cells were stained with dansylcadaverine (MDC; SolarBio), which is designed to observe cellular morphology and autophagy using fluorescence microscopy. MDC is a fluorescent, eosinophilic chromatin pigment that is commonly used to detect autophagy. The methodology of the cell autophagy detection kit was suitable for autophagy staining of cultured cells, and the MDC staining solution could also be combined 
with EB for double staining. The activated filter wavelength was $355 \mathrm{~nm}$, and the blocking filter wave length was $512 \mathrm{~nm}$.

Real-time quantitative PCR on miR-2954 and autophagy and apoptosis related genes mRNA levels

For qRT-PCR quantification of miR-2954 and the mRNA of target genes, miRNA and total RNA were isolated from myocardial tissues and cultured cardiomyocytes. Reverse transcription was performed using a miRcute microRNA FirstStrand cDNA Synthesis Kit (Tiangen Biotech Co. Ltd., Beijing) according to the manufacturer's instructions (Roche). The primers are shown in Table 1 that were used in qRT-PCR for the detection of miR-2954 and target mRNAs related to the PI3K pathway in autophagy and apoptosis, which were the following: IRS, PI3K, AKT, RAS, MEK1, MEK2, ERK, TSC1, TSC2, mTOR, ULK1, ATG13, LKB1, BECLIN1, LC3, BCL2, Caspase3, Caspase8, Caspase9, Cytc, BAX, BAK, P53, JNK, TNF and GAPDH. GAPDH and U6 were used as the housekeeping gene for mRNAs and miRNAs, respectively. qRT-PCR was performed using the Light Cycler $^{\circledR} 480$ System (Roche, Basel, Switzerland) and Fast Universal SYBR Green Master Mix (Roche, Basel, Switzerland). Only one peak for each PCR product was shown in the melting curve analysis. The $2^{-\Delta \Delta \mathrm{ct}}$ method was used to calculate the relative abundance of mRNAs and the miRNA, accounting for genespecific efficiencies and normalized to the mean expression of the above mentioned index.

Western blot analysis of autophagy and apoptosis-related proteins

Total protein was assessed via SDS-polyacrylamide gel electrophoresis under reducing conditions on $12 \%$ gels and then was transferred to nitrocellulose membranes via tank transfer at $80 \mathrm{mV}$ in Tris-glycine buffer containing $20 \%$ methanol for $12 \mathrm{~h}$ at $4^{\circ} \mathrm{C}$. Nitrocellulose membranes were blocked for $1 \mathrm{~h}$ with $5 \%$ bovine serum albumin (BSA) at $37^{\circ} \mathrm{C}$ and were incubated for $12 \mathrm{~h}$ with the following antibodies at $4^{\circ} \mathrm{C}$ : PI3K (1:500, Immuno Way, China), AKT (1:500, Immuno Way, China), mTOR (1:500, Immuno Way, China), LC3 (1:1000, Immuno Way, China), Beclin1 (1:500, ImmunoWay, China), BCL2 (1:500, ImmunoWay, China), BAX (1:500, ImmunoWay, China), Caspase3 (1:500, ImmunoWay, China), Caspase8 (1:500, ImmunoWay, China). PBST was used to wash the nitrocellulose membranes 4 times, for 15 minutes each time. Next, the membranes were incubated with horseradish peroxidase-conjugated secondary antibody against rabbit IgG (1:5000, Santa Cruz, CA, USA) using an ECL kit (Kangweishiji Biotechnology, Beijing, China). Then nitrocellulose membranes were washed 4 times using PBST, each time for 15 minutes. The GAPDH content was analyzed as the loading control with rabbit polyclonal antibody (Sigma, USA).

\section{Flow cytometry analysis of apoptosis}

An Annexin V-FITC/PI apoptosis detection kit (Solarbio, China) was used to treat the transfected cardiomyocytes according to the manufacturer's instructions. Live cells were quantitated via flow cytometry analysis of cells negative for DAPI (4, 6-diamidino-2-phenylindole). For apoptosis detection, cardiomyocytes were incubated with $5 \mu \mathrm{L}$ of Annexin V-FITC and $5 \mu \mathrm{L}$ of PI in $1 \mathrm{~mL}$ of binding buffer for 30 min in the dark. After incubation, apoptotic, necrotic and live cell populations were detected by flow cytometric analysis. 
Statistical analyses

Statistical analyses were performed using GraphPad Prism 5.0 software (version 5.0, Graph Pad Software Inc., San Diego, CA, USA). All the data were analyzed by Student's t-test or one-way ANOVA after a normal distribution was determined and equal variance testing was passed. Quantitative data are presented as the mean \pm SD. Expression of related proteins pictures were cut using Adobe Photoshop CS6 (version CS6, Adobe, USA), and ImageJ (version 1.48, National Institutes of Health, USA) was used to analyze the gray level. Different superscript letters in figure images represent significant differences $(\mathrm{P}<0.05)$.

\section{Results}

\section{Se-deficient model successfully established}

Chickens in the low-Se diet group were exhibited typical myocardial damage, which was previously described by our group and is widely accepted by relevant scholars and researchers. Thus, the Se-deficient model was successfully established.

\section{MicroRNAome analysis and target gene prediction}

Based on the microRNAome results, we detected 11 miRNAs, 7 that were up regulated and 4 that were down-regulated, in Se-deficient cardiac tissues compared with control group (Fig. 1A). miR-2954 was up-regulated to a level 3 -fold higher than that in the control group (Fig. 1B). Target genes were chosen by cross-checking miRDB and TargetScan databases. Then, qRT-PCR was used to further determine the expression of miR-2954 and related genes; the results were consistent with the microRNAome results. Finally, the miR2954 target gene PI3K was further confirmed with a luciferase activity assay.

Ultrastructural observation showed cardiac injury

The control group exhibited normal cardiac muscle morphology with numerous mitochondria and prominent myofilaments (Fig. 1C), and no ultrastructural changes were observed. By contrast, electron microscopic observations of the Sedeficient group showed profound mitochondrial damage and vacuolation of the cytoplasmic reticulum,

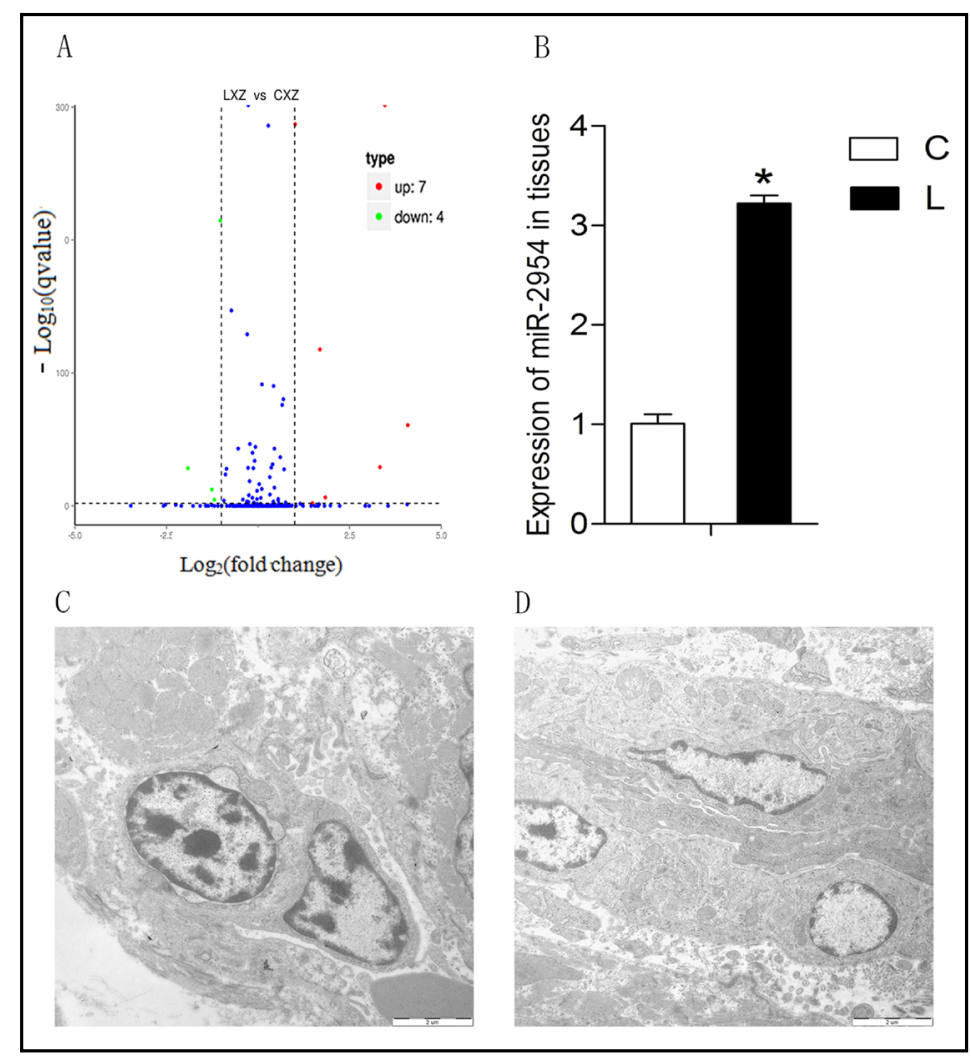

Fig. 1. MicroRNAome analysis, miRNA expression profiles and electron microscopy sections. (A) Red indicates up-regulated miRNAs, and green indicates down-regulated miRNAs compared with the control group. (B) Differential expression of selected miRNA miR2954 in control and Se-deficient tissues. C represents the control group and L is short for low selenium group. (C) Electron microscopy photo of a heart from the control group. (D) Electron microscopy photo of a heart from the selenium-deficient group. 
which develops as a result of myocardial cell autophagy and is a typical feature of apoptosis when cellular atrophy occurs in cardiomyocytes (Fig. 1D).

\section{Cardiomyocytes culture and transfection}

We successfully transfected cultured cardiomyocytes with miR-2954 mimic, mimic $\mathrm{NC}$, inhibitor, and inhibitor NC at different concentrations, and the optimal transfection concentrations were determined (Fig. 2A), which further demonstrated the success of cardiomyocyte transfection.

\section{Luciferase activity assay confirmed PI3K is a target gene of miR-2954}

Based on the cultured transfected cardiomyocytes and to accurately predict target genes, we used a 3'UTR-mediated luciferase activity assay and found that the miR-2954 mimic markedly modulated the luciferase activity driven by PI3K mRNA 3'UTR plasmids. However, the miR-2954 mimic with a mutated target sequence failed to inhibit the luciferase activity driven by PI3K mRNA 3'UTR plasmids (Fig. 2B). These results demonstrate that PI3K is a specific downstream target gene of miR-2954 and might participate in cardiac autophagy induced by Se deficiency.

\section{RNA-Protein Interaction Prediction and Molecular docking analysis verified PI3K as target gene}

RNA-Protein Interaction Prediction (RPISeq) showed that the interaction probability using the RF classifier was 0.55 and that using the SVM classifier was 0.82 (Fig. 3A). Hex8.0.0 software showed that the best docking mode between miR-2954 and PI3K generated 112.9 $\mathrm{kJ}$ of heat. The best docking model is shown in Fig. 3B. We found that the PyMOL software best illustrated the binding between miR-2954 and PI3K (Fig. 3C-3D).

mRNA expression of genes related to autophagy increased in miR-2954 mimic group and selenium deficient group

The qRT-PCR results of the in vivo study showed that the level of miR-2954 in the Sedeficient group was 3.5-fold higher than that in cardiac tissue from the control group, which further verified the predicted results (Fig. 2A). The primers used for detection of miR2954 and the target mRNAs related to the PI3K pathway are listed in Table 1 and Table 2. The expression of IRS, AKT, RAS, MEK1, MEK2, ERK, TSC1, TSC2, mTOR, ULK1, ATG13, LKB1, BECLIN1, and LC3 was detected in cells, and the miR-2954 mimic up-regulated TSC1, TSC2, ULK1, ATG13, LKB1, BECLIN1 and LC3, whereas the other proteins were down-

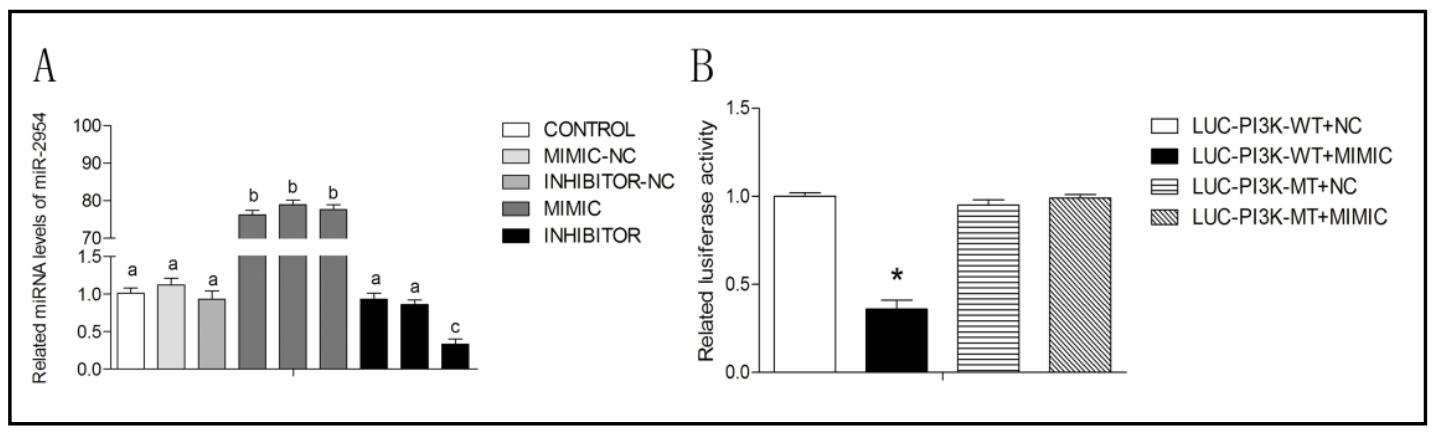

Fig. 2. Detection of the optimal cardiomyocyte tranfection concentration and luciferase activity assay results. (A) Investigation of the most appropriate concentration for $24 \mathrm{~h}$ cardiomyocyte transfection with miR-2954-mimic, miR-2954-inhibitor, mimic negative control and inhibitor negative control. miRNA levels of miR-2954 was determined by qRT-PCR. The data are expressed as the mean \pm SD of triplicate cell cultures. (B) Correlation between PI3K and miR-2954 based on luciferase reporter gene assay results. Bars that do not share the same letters are significantly different $(p<0.05)$. The data are expressed as the mean \pm SD of triplicate cell cultures. 


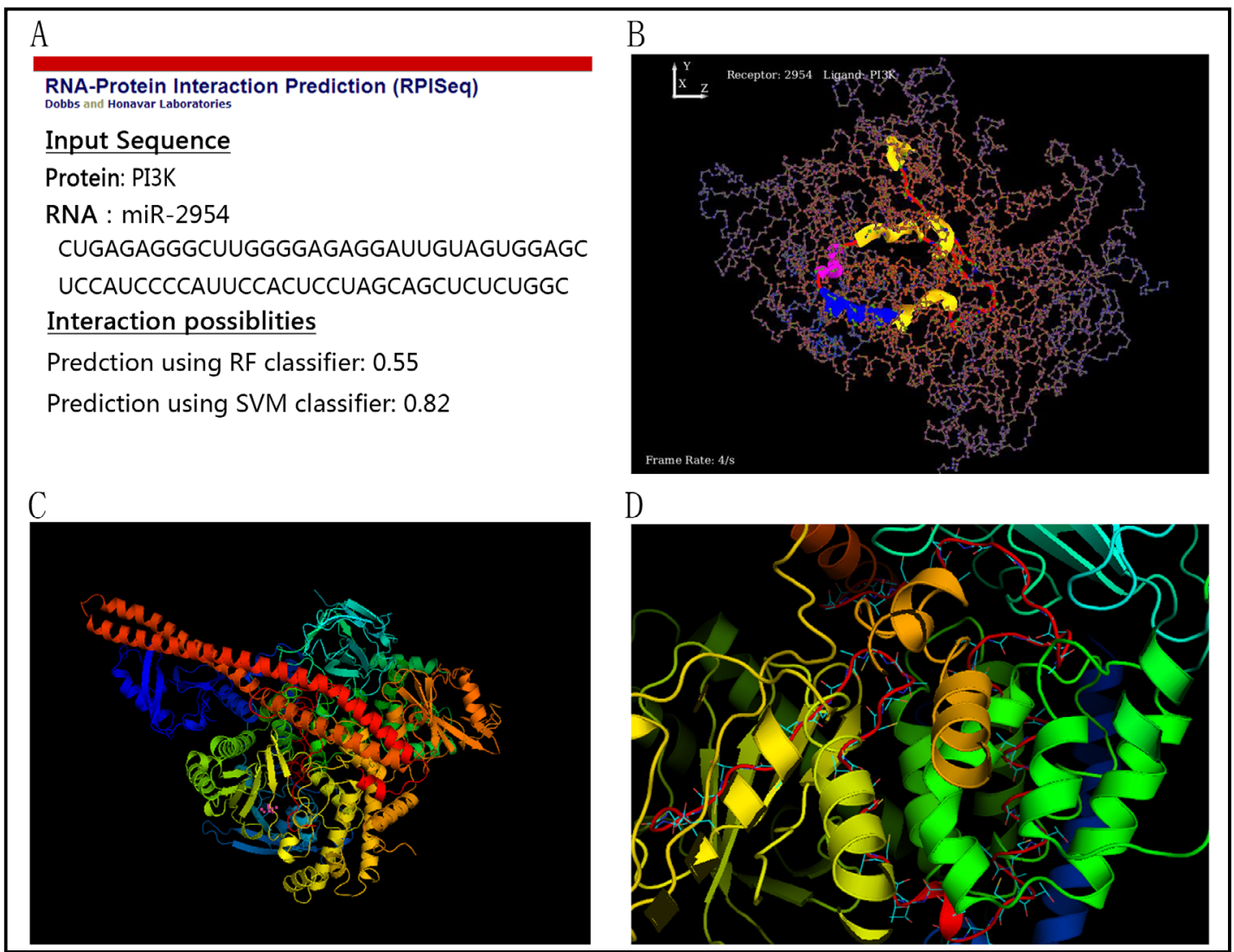

Fig. 3. RNA-Protein Interaction Prediction (RPISeq) and Molecular docking analysis. (A) RPISeq results showed that interaction probabilities. (B) The best docking model is showed in Fig. 3B. miR-2954 was shown in yellow ,blue and pink band; and the PI3K carbon skeleton was shown with balls and sticks. (C) The color band in the center of Fig. 3C was 3D structure of PI3K; however, miR-2954 couldn't be shown at this angle. (D) Fig. 3D showed a magnification of another angle of the model presented in Fig. 3C. The central pink blue chain with branches showed the position of miR-2954 while binding to PI3K.

regulated. Inhibition of miR-2954 resulted in down-regulated expression of TSC1, TSC2, ULK1, ATG13, LKB1, BECLIN1 and LC3, with the expression of other related mRNAs upregulated. We obtained similar results for mRNA expression in the control group and Sedeficient group tissues, which further verified our prediction regarding the inhibitory role of miR-2954 in autophagy. The mRNA expression levels in tissues and cardiomyocytes are shown in Fig. 4A and Fig. 4B, respectively.

mRNA expression of apoptosis-related genes increased in miR-2954 mimic group and selenium deficient group

The primers used for detection of miR2954 and the target mRNAs related to the PI3K pathway are listed in Table 1 and Table 2. The expression of BCL2, Caspase3, Caspase8, Caspase9, Cytc, BAX, BAK, P53, JNK,
Table 2. Sequences of primers used in present research

\begin{tabular}{|c|c|}
\hline Primer & Sequence \\
\hline \multirow{2}{*}{ BCL2 } & Forward: 5'- ATCGTCGCCTTCTTCGAGTT - $3^{\prime}$ \\
\hline & Reverse: 5' - ATCCCATCCTCCGTTGTTCT - $3^{\prime}$ \\
\hline \multirow{2}{*}{ JNK } & Forward: 5'- CAGATAAGCAGTTAGATGAGAG - $3^{\prime}$ \\
\hline & Reverse: $5^{\prime}$ - GACAGATGACGACGAAGAT $-3^{\prime}$ \\
\hline \multirow{2}{*}{ BAX } & Forward: 5' - TCCATTCAGGTTCTCTTGACC $-3^{\prime}$ \\
\hline & Reverse: 5'- GCCAAACATCCAAACACAGA -3' \\
\hline \multirow{2}{*}{ BAK } & Forward: $5^{\prime}$ - CACGTCTACCAGCAAGGCATCAC $-3^{\prime}$ \\
\hline & Reverse: 5'- CCAGCACCACCAGCATGTACTTC - $3^{\prime}$ \\
\hline \multirow{2}{*}{ CASPASE3 } & Forward: 5'- CTGAAGGCTCCTGGTTTA $-3^{\prime}$ \\
\hline & Reverse: 5'- TGCCACTCTGCGATTTAC - $3^{\prime}$ \\
\hline \multirow{2}{*}{ CASPASE8 } & Forward: $5^{\prime}$ - CCCTGAAGACAGTGCCATTT $-3^{\prime}$ \\
\hline & Reverse: 5'- GGGTCGGCTGGTCATTTTAT -3' \\
\hline \multirow{2}{*}{ CASPASE9 } & Forward: 5'- ATTCCTTTCCAGGCTCCATC $-3^{\prime}$ \\
\hline & Reverse: 5' - CACTCACCTTGTCCСTCCAG - $3^{\prime}$ \\
\hline \multirow{2}{*}{ TNF } & Forward: 5'- AGGATAGCGGCACACCTGACG -3' \\
\hline & Reverse: 5'- GTGGCCTCTCCTCGATGACTCC $-3^{\prime}$ \\
\hline \multirow{2}{*}{ C-YTC } & Forward: $5^{\prime}$ - TGGACCTGAAGCGACCTCTGC $-3^{\prime}$ \\
\hline & Reverse: $5^{\prime}$ - CATCACATCTTCACGACGGTAGGC $-3^{\prime}$ \\
\hline \multirow{2}{*}{ GAPDH } & Forward: 5'- AGAACATCATCCCAGCGT - $3^{\prime}$ \\
\hline & Reverse: 5' - AGCCTTCACTACCCTCTTG -3' \\
\hline miR-2954 & 5'-САТССССАТТССАСТССТАG-3' \\
\hline U6 & 5'-CACGCAAATTCGTGAAGCGTTCCA-3' \\
\hline
\end{tabular}


and TNF was detected in cells, and the miR-2954 mimic up-regulated Caspase3, Caspase8, Caspase9, Cytc, BAX, BAK, P53, JNK, and TNF, but BCL2 was down-regulated by the miR2954 mimic. Inhibition of miR-2954 down-regulated the expression of Caspase3, Caspase8, Caspase9, Cytc, BAX, BAK, P53, JNK, and TNF, with the expression of BCL2 up-regulated. We obtained similar results for mRNA expression in the control group and Se-deficient group tissues, which further verified our prediction regarding the inhibitory role of miR-2954 in apoptosis. The expression of mRNAs in tissues and cardiomyocytes is shown in Fig. 5A and Fig. 5B, respectively.

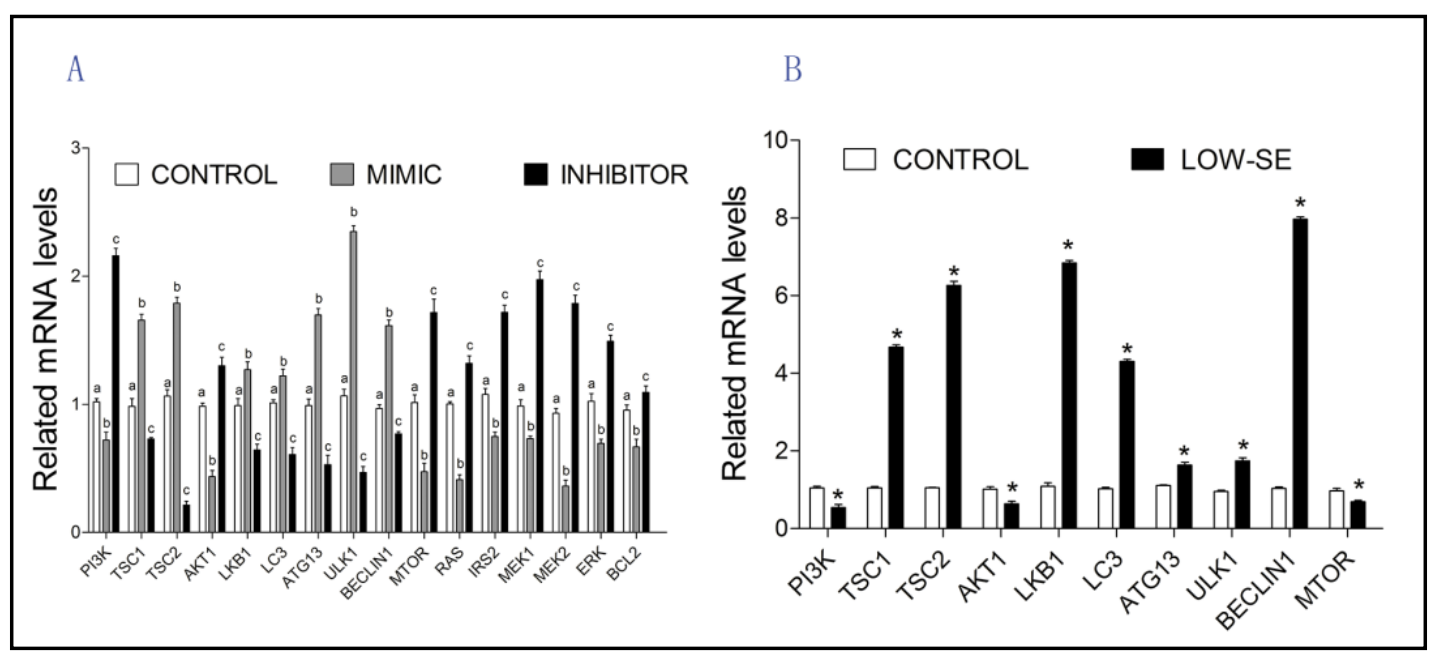

Fig. 4. Different mRNA expression levels of PI3K and autophagy-related genes in vivo and in vitro. (A) The mRNA levels in cardiomyocytes transfected with mimic and inhibitor and in control cardiomyocytes. Bars represent the mean \pm SD of triplicate cell cultures. Bars that do not share the same letters are significantly different $(\mathrm{p}<0.05)$ from each other. (B) The mRNA expression in control and Se-deficient myocardial tissue. $\mathrm{b}$ and $\mathrm{c}$ are significantly different from $\mathrm{a}$; b is the mimic group, and $\mathrm{c}$ is the inhibitor group; *indicates a significant difference compared with the control group.

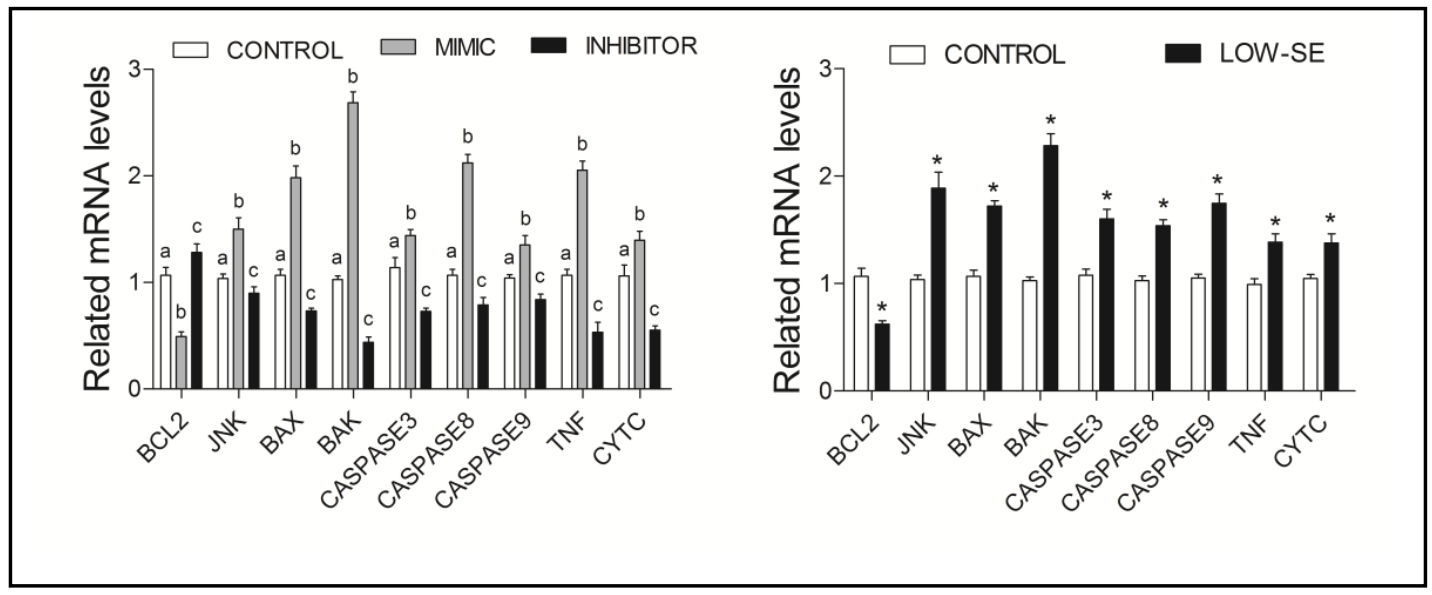

Fig. 5. Different PI3K and apoptosis-related genes mRNA expression levels in vivo and in vitro. (A) The mRNA expression in cardiomyocytes transfected with mimic, inhibitor or negative control. (B) The mRNA levels in the control and Se-deficient myocardial tissues. Bars represent the mean \pm SD of 30 individuals. ${ }^{*} \mathrm{p}<0.05$ by Student's t-test. 
Western blot analysis of autophagy-related proteins confirmed autophagy increased in miR-2954 mimic group and selenium deficient group

After detecting the expression of mRNA levels of the PI3K pathway, we further validated our prediction at the protein level. Knockdown of miR-2954 by the miR-2954inhibitor and overexpression of miR-2954 by the miR-2954mimic were used. Additionally, we tested the protein levels of PI3K, AKT, mTOR, BECLIN1, LC3 and GAPDH in transfected cardiomyocytes. The results for autophagy proteins in tissues and cardiomyocytes are shown in Fig. 6A and Fig. 6B, respectively. The results for cardiomyocytes transfected with the mimic showed that expression of LC3 and BECLIN1 was up-regulated, with a decrease in PI3K, AKT and mTOR. We observed opposite results, when miR-2954 was inhibited in cardiomyocytes. Additionally, in Se-deficient

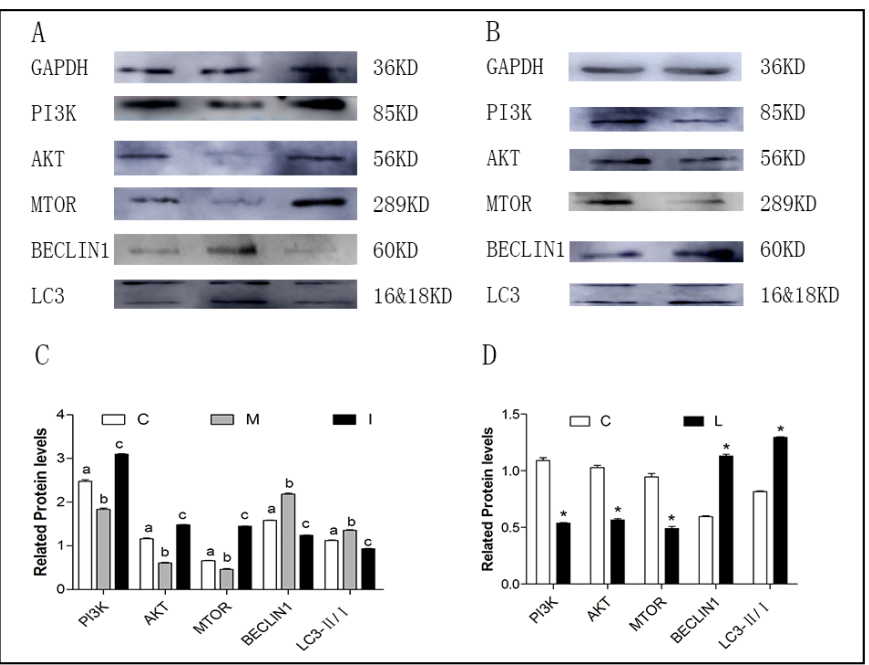

Fig. 6. Levels of autophagy-related proteins in vivo and vitro. (A) The protein levels of GAPDH, AKT, PI3K, mTOR, LC3 and Beclin1 were detected in vitro. (B) In vivo autophagy related proteins in control group and selenium deficient group were shown from left to right respectively. Bars represent the mean \pm SD. Bars that do not share the same letters are significantly different $(p<0.05)$ from each other. ${ }^{*} \mathrm{p}<0.05$ by Student's t-test. (C) Differential expression of autophagy associated proteins in control, mimic and inhibitor groups by histogram. (D) Differential expression of autophagy associated proteins in control and selenium deficient groups by histogram. $\mathrm{C}$ indicates the control group; $\mathrm{M}$ indicates the mimic group; I indicates inhibitor group. tissues, the levels of PI3K, AKT and mTOR decreased as the levels of the other proteins were up-regulated, compared with tissues from the control group. In vivo and in vitro experiments showed that the protein levels were consistent with mRNA levels. Combined, these results further demonstrated in vivo and in vitro that PI3K is the target gene of miR-2954, and these findings demonstrated their inhibitory role in autophagy generation.

Western blot analysis of apoptosis-related proteins confirmed apoptosis increased in miR2954 mimic group and selenium deficient group

We examined the protein level of Caspase3, Caspase8, BAX, and BCL2 in transfected cardiomyocytes. The autophagy-related proteins levels in tissues and cardiomyocytes are shown in Fig. 7A and Fig. 7B, respectively. Cardiomyocytes transfected with the mimic exhibited up-regulated expression of Caspase3, Caspase8 and BAX was up regulated, and decreased BCL2 expression. We observed the opposite results, when miR-2954 was inhibited in cardiomyocytes. Additionally, in Se-deficient tissues, the levels of Caspase3, Caspase 8 and BAX were increased and the level of BCL2 was decreased, compared with tissues from the control group. In vivo and in vitro experiments showed that the protein levels were consistent with the mRNA levels.

Fluorescence detection of autophagy in cardiomyocytes confirmed autophagy increased in miR-2954 mimic group

We found that the tinctorial yield in cardiomyocytes transfected with the miRNA mimic was clearly much higher than that in inhibitor and control group of cardiomyocytes, which exhibited obvious autophagy features. Compared with the control group, the inhibitor group showed weaker fluorescence; by contrast, cardiomyocytes transfected with mimic showed obvious fluorescent spots that indicated autophagy (Fig. 8). 
Liu et al.: Role of miR-2954 in Se Deficiency Myocardium

Flow cytometry analysis of apoptosis confirmed apoptosis increased in miR-2954 mimic group

The delta quadrant in the flow cytometry analysis result reflects the number of apoptotic cells, which further show indicates the percentage of apoptotic cardiomyocytes. In Fig. 9B, apoptosis was obviously increased in cardiomyocytes transfected with that in the mimic compared with that in the control and inhibitor groups, as shown in Fig. 9A and 9C, respectively. In addition,the percentage of apoptotic cells in cardiomyocytes transfected with the inhibitor was lower than that in the control group, further demonstrating the role of miR-2954 in apoptosis.

Fig. 7. Levels of apoptosis-related proteins in vivo and vitro. (A) From left to right, was control group, mimic group, inhibitor group in cardiomyocytes, respectively. (B) In vivo apoptosis-related proteins in control group and selenium deficient group were shown from left to right. Bars represent the mean \pm SD. Bars that do not share the same letters are significantly different $(p<0.05)$ from each other.* $\mathrm{p}<0.05$ by Student's t-test. (C) Differential expression of apoptosis associated proteins in control, mimic and inhibitor groups by histogram. (D) Differential expression of apoptosis associated proteins in control and selenium-deficient group is shown in histogram.
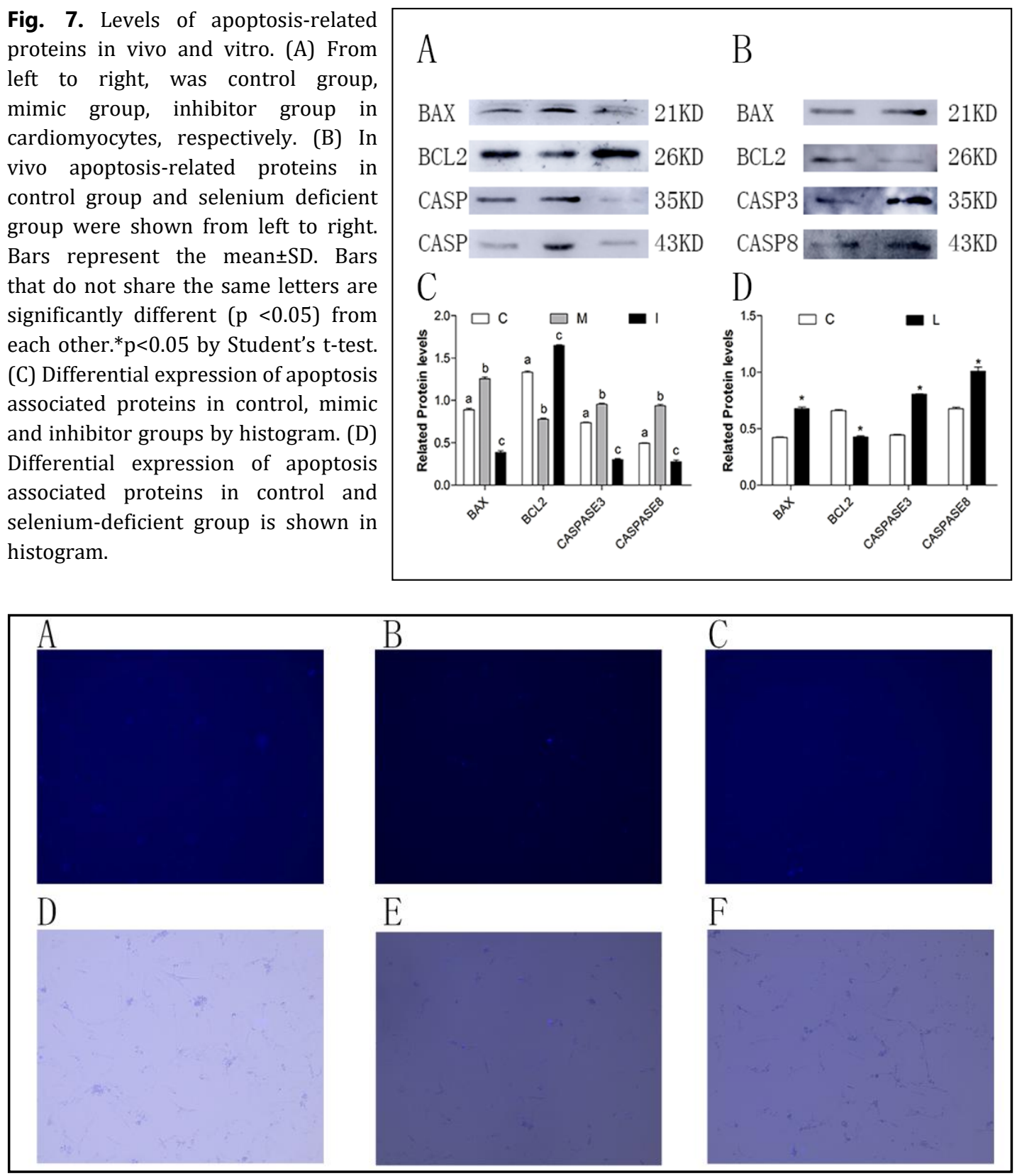

Fig. 8. Detection of autophagosomes via fluorescence detection in cardiomyocytes. (A-C) Fluorescent staining of cardiomyocytes transfected with control, mimic or inhibitor; fluorescence signals are shown in blue. (D-F) These three images further show position of fluorescent puncta in cardiomyocytes. 


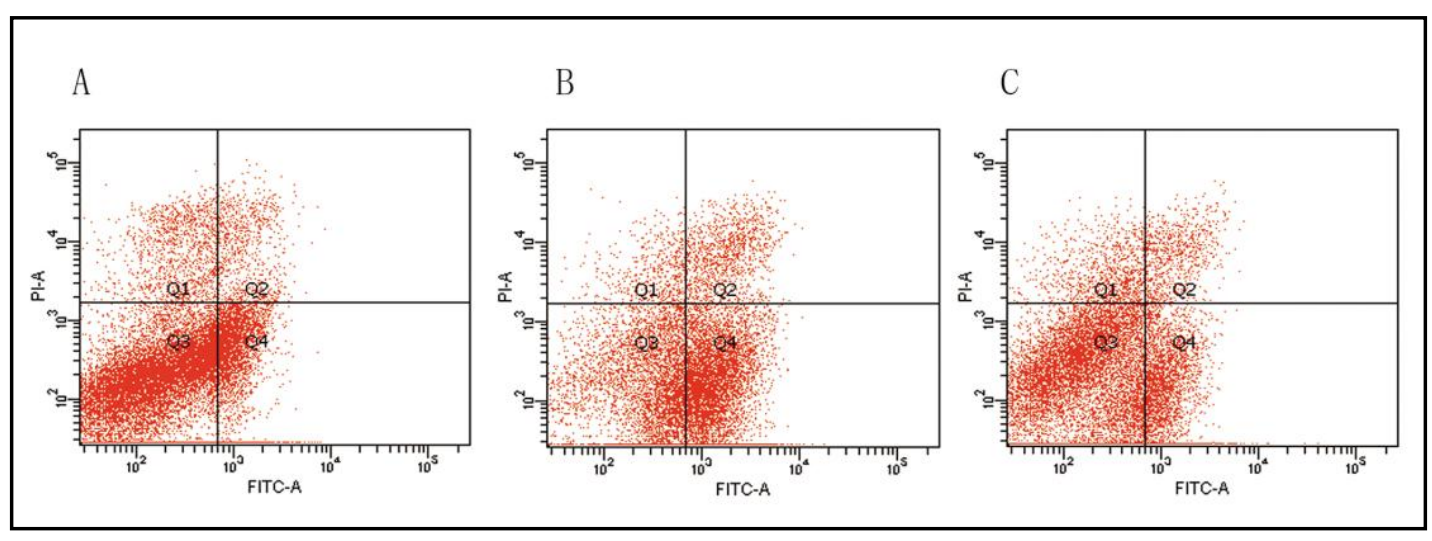

Fig. 9. Evaluation of miR-2954-mediated modulation of PI3K-mediated apoptosis using flow cytometry. Cardiomyocytes were transfected with negative control (A), mimic (B) or inhibitor (C) for $24 \mathrm{~h}$.

\section{Discussion}

The micronutrient selenium is an essential trace element in mammals for physiological health, including in humans. Selenium is incorporated into proteins via the rare amino acid selenocysteine (Sec), and this incorporation occurs during that protein translation $[14,15]$. Selenium and selenoproteins may participate extensively in key biological processes, such as protein folding, cellular differentiation, cellular response to oxidative stress, and the immune response.

Selenium deficiency leading to fatal cardiomyopathy is well known in pigs and in humans and is referred to as MHD and KD, respectively. MHD was described for the first time in 1967 [16]. Additionally, white muscle disease can occur in multiple species, including cattle [17], pigs, horses, sheep, deer, and poultry. Our previous research showed that in chicken, Se deficiency could lead to injury of the myocardium and other pathological changes [18]. In this study, we investigated the mechanism of myocardial injury induced by Se deficiency.

In recent years, miRNAs, which are short chain noncoding RNAs, have been found to play essential roles in heart disease and have received widespread attention. Through microRNAome analysis in humans, Ikeda et al. verified 87 miRNAs that showed significant expression levels in diseases associated with heart failure [19]. Indeed, known pro-fibrotic miRNAs (miR-21 and miR-208) may play an important role in ischemia/reperfusion injury in cardiomyocytes. Pathological overexpression of miR-25 can cause impaired calcium transport and cardiac contractility during severe heart failure in mice and humans, followed by further declining cardiac function [20]. In this study, for the first time, we detected an important miRNA that showed significant expression levels in a Se-deficient chicken model via microRNAome analysis and confirmed that PI3K was the downstream target gene of miR-2954 in vivo and in vitro. miR-2954 was selected for further study from 11 miRNAs detected in the microRNAome, because among these miRNAs, the expression of miR-2954 showed the most significant overall difference. The findings of this study may lead to a better understanding of the mechanisms and miRNA pathways that regulate autophagy-related myocardial disease.

Autophagy is not only an adaptive response of living organisms to nutrient limitation but also a mechanism for cell suicide and is a highly conserved cellular mechanism. Under certain conditions, including starvation or stress $[21,22]$, autophagy represents an adaptive reaction that clears damaged organelles to promote survival under the stress condition, including nutritional bioenergy depletion. However, in other settings, autophagy deregulation may contribute to the development of disease, such as infectious diseases [23], neurodegenerative disorders [24, 25], cancer, or cardiac disease [26]. Under stress and other adverse conditions, apoptosis and autophagy are two well-known pathways that lead to programmed cell death [27]. Apoptosis is a cell death mechanism characterized

\section{KARGER}


by DNA fragmentation, cellular atrophy and formation of apoptotic bodies that may be activated either through the Tumor Necrosis Factor or the Fas pathway or mediated by mitochondrial Cytochrome-C release. Se-deficiency-induced apoptosis has been observed in cardiomyocytes of suckling mice, in chicken liver caused by oxidative-endoplasmic reticulum stress, and in chicken immune organs caused by oxidative stress and nitric oxide. Our present work demonstrated the mechanisms by which miR-2954 and its target gene PI3K regulate autophagy and apoptosis via the PI3K and mTOR pathways.

PI3K is a bridge molecule that links extracellular signaling and the cellular response and can be regulated by upstream signaling molecules and bypass molecules, further influencing a series of downstream signaling molecules to participate in regulation of cell apoptosis and autophagy. Hawkins PT confirmed that inhibition of Class I PI3Ks may offer a therapeutic opportunity to treat nonresolving inflammatory pathologies in humans [28]. Wang et al. demonstrated that TSC1 was frequently down regulated in high-grade serous ovarian carcinoma (HGSOC), and a low TSC1 expression level was associated with advanced tumor stage [29]. ULK1 is a key component in the autophagy pathway. Zheng et al. found that miR26a-5p might regulate autophagy in cardiac fibroblasts by targeting ULK1, which may have an effect on cardiac fibrosis [30]. mTOR can be activated under cell starvation, nutritional deficiency and cellular stress conditions. Additionally, the autophagy gene BECLIN1 binds to PI3K and Vps34 and forms a complex that phosphorylates phosphatidylinositol to produce PIP3, which plays an important role in the formation of the autophagy precursor complex and the origin of the autophagosome membrane. In the present research, we used TargetScan to predict possible target genes of miR-2954, and based on primary screening and pathway analysis of predicted genes, $\mathrm{PI} 3 \mathrm{~K}$, which exhibited the highest score, was selected as the target gene. Then, luciferase activity, qRT-PCR, western blotting, and flow cytometry analyses were used to verify our prediction; finally, PI3K was confirmed to be a target gene of miR-2954. In this study, the mechanism of myocardial injury induced by selenium deficiency in vivo and in vitro was described in detail. However, certain limitations remain; for example, we did not establish whether miR-2954 is overexpressed in chickens or chicken embryos, and the lack of selenium in the cardiomyocyte model was not successfully established. In the future, we intend to establish a model of miR-2954 overexpression in chicken or chicken embryos and study the effect of selenium deficiency using the model.

\section{Conclusion}

In summary, our work revealed that differential miR-2954 expression was significant in a selenium-deficient chicken model and in cardiomyocytes. Additionally, this study is the first to verify that PI3K, an essential component of the PI3K pathway linked with the mTOR pathway, is a target gene of miR-2954, and mTOR-ULK1 is widely known to be involved in autophagy in cardiomyocytes and animal models. Additionally, apoptosis was found to be increased in Se-deficient tissues and in cardiomyocytes transfected with miR-2954 mimics. In conclusion, miR-2954 regulates myocardial autophagy and apoptosis induced by Se deficiency by targeting the PI3K pathway. In our study, during the selenium-induced cardiac injury process, autophagy and apoptosis showed synergistic activity. Accordingly, a better understanding of miR-2954 regulation may provide new insights into the development and treatment of heart disease caused by Se deficiency and lead to treatments for cardiac diseases related to autophagy.

\section{Acknowledgements}

This study was supported by the National Natural Science Foundation of China (31872531), Open Projects of Key Laboratory of Animal Cellular and Genetic Engineering of Heilongjiang Province (KF201703), Merit-based Funding for Returned Oversea Student

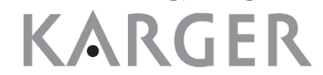




\section{Cellular Physiology Cell Physiol Biochem 2018;51:778-792 and Biochemistry Published \begin{tabular}{l|l} 
DOI: 10.1159/000495332 2018 The Author(s). Published by S. Karger AG, Basel \\
wisw.karger.com/cpb
\end{tabular}}

Liu et al.: Role of miR-2954 in Se Deficiency Myocardium

of Heilongjiang Province (2018QD0005), Foundation for Young Talents in Higher Education of Heilongjiang, China (UNPYSCT-2015009), "Academic backbone" Project of Northeast Agricultural University (17XG11).

\section{Disclosure Statement}

The authors have no conflicts of interest to declare.

\section{References}

-1 Liu Q, Yang J, Cai J, Luan Y, Sattar H, Liu M, Xu S, Zhang Z: Analysis of the Interactions Between Thioredoxin and 20 Selenoproteins in Chicken. Biol Trace Elem Res 2017;179:304-317.

-2 Jablonska E, Reszka E, Gromadzinska J, Wieczorek E, Krol MB, Raimondi S, Socha K, Borawska MH, Wasowicz W: The Effect of Selenium Supplementation on Glucose Homeostasis and the Expression of Genes Related to Glucose Metabolism. Nutrients 2016;8:772-784.

-3 Klein EA, Thompson I, Tangen CM, Lucia MS, Goodman P, Minasian LM, Ford LG, Parnes HL, Gaziano JM, Karp DD, Lieber MM, Walther PJ, Parsons JK, Chin J, Darke AK, Lippman SM, Goodman GE, Meyskens FL, Baker LH: Vitamin E and the risk of prostate cancer: Updated results of the Selenium and Vitamin E Cancer Prevention Trial (SELECT). J Clin Oncol 2012;30:7-15.

-4 Yao HD, Wu Q, Zhang ZW, Li S, Wang XL, Lei XG, Xu SW: Selenoprotein W serves as an antioxidant in chicken myoblasts. Biochim Biophys Acta 2013;1830:3112-3120.

-5 Zhang ZW, Wang QH, Zhang JL, Li S, Wang XL, Xu SW: Effects of oxidative stress on immunosuppression induced by selenium deficiency in chickens. Biol Trace Elem Res 2012;149:352-361.

-6 Wang R, Sun B, Zhang Z, Li S, Xu S: Dietary selenium influences pancreatic tissue levels of selenoprotein W in chickens. J Inorg Biochem 2011;105:1156-1160.

7 Yu J, Yao H, Gao X, Zhang Z, Wang JF, Xu SW: The role of nitric oxide and oxidative stress in intestinal damage induced by selenium deficiency in chickens. Biol Trace Elem Res 2015;163:144-153.

-8 Yang J, Zhang Y, Hamid S, Cai J, Liu Q Li H, Zhao R, Wang H, Xu S, Zhang Z: Interplay between autophagy and apoptosis in selenium deficient cardiomyocytes in chicken. J Inorg Biochem 2017;170:17-25.

-9 Loscalzo J: Keshan Disease, Selenium Deficiency, and the Selenoproteome. New England Journal of Medicine 2014;370:1756-1760.

10 Zhang M, Wei J, Yan R, Shan H, Pan X: ASSA13-03-32 The Alterations of Heart Function and Cardiac Mitochondria in Selenium Deficiency Rats. Heart 2013;99:A24-A24.

-11 Jeong D, Yoo J, Lee P, Kepreotis SV, Lee A, Wahlquist C, Brown BD, Kho C, Mercola M, Hajjar RJ: miR-25 Tough Decoy Enhances Cardiac Function in Heart Failure. Mol Ther 2018; 26(3):718-729.

-12 Verjans R, Peters T, Beaumont F, Van RL, Van TH, Verhesen W, Munts C, Bijnen M, Henkens M, Diez J: MicroRNA-221/222 Family Counteracts Myocardial Fibrosis in Pressure Overload-Induced Heart Failure. Hypertension 2017; 71(2):280-288.

13 Yang T, Cao C, Yang J, Liu T, Lei XG, Zhang Z, Xu S: miR-200a-5p regulates myocardial necroptosis induced by Se deficiency via targeting RNF11. Redox Biology 2018;15:159-169.

-14 Kiełczykowska M, Musik I, Kurzepa J, Żelazowska R, Lewandowska A, Paździor M, Kocot J: The Influence of Lithium and/or Selenium Treatment on Homeostasis of Chosen Bioelements in Rats. Biological Trace Element Research 2017;178:1-7.

-15 Papp LV, Lu J, Holmgren A, Khanna KK: From selenium to selenoproteins: synthesis, identity, and their role in human health. Antioxid Redox Signal 2007;9:775-806.

-16 Seffner W, Wittig W, Rittenbach P: Investigations on Mulberry heart disease (microangiopathy) of pigs. Dtsch Tierarztl Wochenschr 1967;74:213-220.

17 Abutarbush SM, Radostits OM: Congenital nutritional muscular dystrophy in a beef calf. The Canadian veterinary journal $=$ La revue veterinaire canadienne 2003;44:738-739.

18 Yang J, Hamid S, Cai J, Liu Q, Xu S, Zhang Z: Selenium deficiency-induced thioredoxin suppression and thioredoxin knock down disbalanced insulin responsiveness in chicken cardiomyocytes through PI3K/Akt pathway inhibition. Cell Signal 2017;38:192-200. 


\section{Cellular Physiology Cell Physiol Biochem 2018;51:778-792

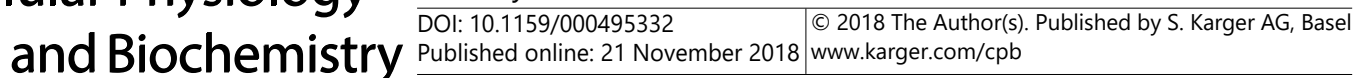

19 Ikeda S, Kong SW, Lu J, Bisping E, Zhang H, Allen PD, Golub TR, Pieske B, Pu WT: Altered microRNA expression in human heart disease. Physiol Genomics 2007;31:367-373.

20 Wahlquist C, Jeong D, Rojasmuñoz A, Kho C, Lee A, Mitsuyama S, Van MA, Park WJ, Sluijter JP, Doevendans PA: Inhibition of miR-25 improves cardiac contractility in the failing heart. Nature 2014;508:531-535.

-21 Levine B, Klionsky DJ: Development by self-digestion: molecular mechanisms and biological functions of autophagy. Developmental Cell 2004;6:463-477.

-22 Lum JJ, DeBerardinis RJ, Thompson CB: Autophagy in metazoans: cell survival in the land of plenty. Nature reviews. Molecular cell biology 2005;6:439-448.

-23 Gutierrez MG, Master SS, Singh SB, Taylor GA, Colombo MI, Deretic V: Autophagy is a defense mechanism inhibiting BCG and Mycobacterium tuberculosis survival in infected macrophages. Cell 2004;119:753-766.

-24 Komatsu M, Waguri S, Chiba T, Murata S, Iwata J, Tanida I, Ueno T, Koike M, Uchiyama Y, Kominami E: Loss of autophagy in the central nervous system causes neurodegeneration in mice. Nature 2006;441:880-884.

-25 Hara T, Nakamura K, Matsui M, Yamamoto A, Nakahara Y, Suzuki-Migishima R, Yokoyama M, Mishima K, Saito I, Okano H, Mizushima N: Suppression of basal autophagy in neural cells causes neurodegenerative disease in mice. Nature 2006;441:885-889.

-26 Rabkin SW: Nitric Oxide-Induced Cell Death in the Heart: The Role of Autophagy. Autophagy 2007;3:347349.

27 Bongaerts GP: What of apoptosis is important: the decay process or the causative origin? Med Hypotheses 2008;70:482-487.

28 Stoyanov B, Volinia S, Hanck T, Rubio I, Loubtchenkov M, Malek D, Stoyanova S, Vanhaesebroeck B, Dhand R, Numberg B: Cloning and characterization of a G protein-activated human phosphoinositide-3 kinase. Science (New York, N.Y.) 1995;269:690-693.

29 Wang Y, Zhang X, Tang W, Lin Z, Xu L, Dong R, Li Y, Li J, Zhang Z, Li X, Zhao L, Wei JJ, Shao C, Kong B, Liu Z: miR-130a upregulates mTOR pathway by targeting TSC1 and is transactivated by NF-kappaB in high-grade serous ovarian carcinoma. Cell Death Differ 2017;24:2089-2100.

30 Zheng L, Lin S, Lv C: MiR-26a-5p regulates cardiac fibroblasts collagen expression by targeting ULK1. Sci Rep 2018;8:2104-2118. 